\title{
Microtubule actin cross-linking factor 1, a novel target in glioblastoma
}

\author{
NAJLAA AFGHANI $^{1}$, TORAL MEHTA ${ }^{1}$, JIALIANG WANG $^{2}$, NAN TANG $^{3}$, \\ OMAR SKALLI $^{4}$ and QUINCY A. QUICK ${ }^{1}$
}

\begin{abstract}
${ }^{1}$ Department of Biological Sciences, Tennessee State University, Nashville, TN; ${ }^{2}$ Department of Neurological Surgery, Vanderbilt Medical Center, Nashville, TN, USA; ${ }^{3}$ Union Hospital, Tongji Medical College, Huazhong University of Science and Technology, Wuhan, Hubei, P.R. China; ${ }^{4}$ Department of Biological Sciences, University of Memphis, Memphis, TN, USA
\end{abstract}

Received October 13, 2016; Accepted December 5, 2016

DOI: $10.3892 /$ ijo.2016.3798

\begin{abstract}
Genetic heterogeneity is recognized as a major contributing factor of glioblastoma resistance to clinical treatment modalities and consequently low overall survival rates. This genetic diversity results in variations in protein expression, both intratumorally and between individual glioblastoma patients. In this regard, the spectraplakin protein, microtubule actin cross-linking factor 1 (MACF1), was examined in glioblastoma. An expression analysis of MACF1 in various types of brain tumor tissue revealed that MACF1 was predominately present in grade III-IV astroctyomas and grade IV glioblastoma, but not in normal brain tissue, normal human astrocytes and lower grade brain tumors. Subsequent genetic inhibition experiments showed that suppression of MACF1 selectively inhibited glioblastoma cell proliferation and migration in cell lines established from patient derived xenograft mouse models and immortalized glioblastoma cell lines that were associated with downregulation of the Wnt-signaling mediators, Axin1 and $\beta$-catenin. Additionally, concomitant MACF1 silencing with the chemotherapeutic agent temozolomide (TMZ) used for the clinical treatment of glioblastomas cooperatively reduced the proliferative capacity of glioblastoma cells. In conclusion, the present study represents the first investigation on the functional role of MACF1 in tumor cell biology, as well as demonstrates its potential as a unique biomarker that can be targeted synergistically with TMZ as part of a combinatorial therapeutic approach for the treatment of genetically multifarious glioblastomas.
\end{abstract}

Correspondence to: Dr Quincy A. Quick, Department of Biological Sciences, Tennessee State University, 3500 John A. Merritt Boulevard, Nashville, TN 37209, USA

E-mail: qquick@tnstate.edu

Abbreviations: MACF1, microtubule actin cross-linking factor 1; TMZ, temozolomide

Key words: glioblastoma, microtubule actin cross-linking factor 1, temozolomide, Wnt signaling

\section{Introduction}

Glioblastomas have an age adjusted incidence of 3 per 100,000 persons per year in the United States and comprise approximately $45 \%$ of all gliomas with a relative survival rate of $5 \%$, 5 -years post-diagnosis (1), which can be attributed in part to high rates of tumor recurrence. A major contributor to the low survival rates of patients with these cancers is the incredibly complex glioblastoma genome, which has a vast number of uncharacterized expressed genetic abnormalities that contribute to intra- and intertumor variation that negatively impact therapeutic response and patient outcomes (2). The poor prognosis of brain tumor patients, particularly those with glioblastoma, can be attributed in part to the limited number of aberrantly expressed factors used clinically as diagnostic and prognostic indicators. These have included genetic mutations, deletions, amplifications, and epigenetic changes in isocitrate dehydrogenase (IDH), EGFR and $\mathrm{O}^{6}$-methylguanine DNA methyltransferase (MGMT) (3-8). In the present study, we investigated microtubule actin cross-linking factor 1 (MACF1), which functions to crosslink microtubules and actin filaments, for its role in tumor cell biology and as a novel target in glioblastoma. Although to date MACF1 has received little consideration in tumor cell biology and as a target in human cancers, its function as a cytoskeleton integrator has been shown to be involved in organization of the cytoskeleton (9), tissue repair (10) and vertebrate development (11). These studies provided correlative evidence that MACF1 is involved in processes such as metastatic invasion in which cytoskeleton organization is a key element that contributes to tumor progression in various human cancers, as well as cellular and physiological processes such as tissue repair when dysregulated in normal tissue can lead to tumorigenesis. Additionally, studies by Sjöblom et al (12) identified MACF1 as a candidate breast cancer gene in a mutational analysis study, while Misquitta-Ali et al (13) detected alternatively spliced exons in MACF1 transcripts from lung cancer patients providing further evidence, albeit circumstantial, that MACF1 plays a role in human cancer. In the present study, we demonstrated that genetic inhibition of MACF1, which we found to be expressed at high levels in glioblastoma relative to normal brain tissue, had anti-tumorigenic effects on glioblastoma 
cell behavior and a synergistic effect with temozolomide, an alkylating agent used clinically to treat this disease. To the best of our knowledge, this is the first investigation to evaluate the functional role of MACF1 in human cancers and as a target that enhances the efficacy of therapeutic treatment modalities.

\section{Materials and methods}

Cells culture conditions and reagents. U251 human glioblastoma cells were purchased from Sigma-Aldrich (St. Louis, MO, USA) and the U87 and A172 human glioblastoma cells were from the American Type Culture Collection (ATCC; Manassas, VA, USA). Normal human astrocytes were obtained from Sciencell Research Laboratories (Carlsbad, CA, USA). All cell lines were maintained in Dulbecco's modified Eagle's medium-DMEM (Invitrogen, Carlsbad, CA, USA) containing $10 \%$ fetal bovine serum (FBS; Invitrogen), $2 \mathrm{mM}$ L-glutamine (Invitrogen), $100 \mathrm{nM}$ MEM non-essential amino acids (Invitrogen) and penicillin-streptomycin (Invitrogen) at $37^{\circ} \mathrm{C}$ and $5 \% \mathrm{CO}_{2}$. Accell SMARTpool siRNAs targeting MACF1 were purchased from GE Dharmacon (Chicago, IL, USA). Temozolomide was purchased from Sigma-Aldrich. GBM76 patient derived xenograft cells were established at the Mayo Clinic (Rochester, MN, USA) (14), while primary xenograft lines T4105 and T4302 were provided by Jeremy Rich from the Cleveland Clinic (Cleveland, OH, USA). Patient derived xenograft cell lines (GBM76, T4105 and T4302) were serially passaged as subcutaneous tumors in immunocompromised mice and maintained as ex vivo cultures in neurobasal medium supplemented with B27 serum substitute, $20 \mathrm{ng} / \mathrm{ml}$ EGF and 20 ng/ml bFGF (Life Technologies, Carlsbad, CA, USA).

Immunohistochemistry. Brain tumor tissue arrays were purchased from US Biomax, Inc., (Rockville, MD, USA) to evaluate MACF1 expression. Each slide contained the following number and array of brain tumor tissue sections provided by the supplier: 10 grade (I-II) astrocytomas, 10 grade (III-IV) astrocytomas, 33 grade (IV) glioblastomas, 3 oligoastrocytomas, 6 grade (I-II) oligodendrogliomas, 3 medulloblastomas, 3 anaplastic oligodendrogliomas, 4 ependymomas and cancer adjacent normal tissue from 5 brains. Paraffin-embedded tissue sections were dewaxed in xylene for $5 \mathrm{~min}$, rehydrated in $100 \%$ ethanol, $95 \%$ ethanol and water. Next tissue was fixed with $100 \%$ methanol at $-20^{\circ} \mathrm{C}$ for $15 \mathrm{~min}$ and washed three times with phosphatebuffered saline (PBS). Detection of MACF1 was conducted using the ImmunoCruz rabbit ABC Staining system (Santa Cruz Biotechnology, Dallas, TX, USA). Tissue sections were blocked for $1 \mathrm{~h}$ in $1.5 \%$ normal horse serum in PBS at room temperature, permeabilized with $0.05 \%$ Triton-X in PBS for $10 \mathrm{~min}$, incubated overnight at $4^{\circ} \mathrm{C}$ with a polyclonal MACF1 antibody (Santa Cruz Biotechnology) and rinsed three times with PBS. Subsequently, sections were incubated with a biotinylated secondary antibody for $3 \mathrm{~h}$ at room temperature and washed in PBS three times 5 min each. Sections were then incubated with an avidin-biotinylated HRP complex for $30 \mathrm{~min}$, rinsed again three times with PBS and MACF1 protein visualized as diaminobenzidine (DAB) positive cells in tissue sections with a peroxidase substrate solution containing substrate buffer, DAB and peroxidase substrate according to the manufacturer's instructions. Images were then taken with a Leica DM4000 microscope and QImaging system (Leica Microsystems, Inc., Buffalo Grove, IL, USA).

MACF1 silencing. For silencing experiments $3.5 \times 10^{4} \mathrm{U} 251$, A172, or patient derived xenograft cells were plated in 6-well plates, grown for two days to $70 \%$ confluency, and treated with $1 \mu \mathrm{M}$ non-targeting control siRNAs, $1 \mu \mathrm{M}$ siRNAs targeting MACF1 (GE Dharmacon), or shRNAs targeting MACF1 from the MISSION shRNA Library (TRCN0000294117 and TRCN0000294123; Sigma-Aldrich) and allowed to incubate for 6 days in Accell media for siRNAs or neurobasal medium for shRNAs. MACF1 expression was subsequently examined in control and MACF1 silenced cells using immunoblotting procedures.

Cell proliferation assay. To assess the effects of silencing MACF1 on cell proliferation and viability, cells were treated with $1 \mu \mathrm{M}$ of non-targeting control siRNAs or siRNAs targeting MACF1 (GE Dharmacon) and allowed to incubate for 6 days in 6 -well plates as described above. At the end of the 6-day incubation period $1.5 \times 10^{4}$ cells treated with control or siRNAs targeting MACF1 were replated in 12-well plates and allowed to incubate in the absence of siRNAs for 2, 4, and 6 days in DMEM media (Invitrogen) containing 10\% FBS (Invitrogen). At the end of each time-point cells were evaluated using the crystal violet cell proliferation assay, one of three standard assays that can be used to evaluate cytotoxicity and/or cell viability that provide a measure of cell proliferation (15). Tissue culture medium was removed and the cell monolayer was fixed with $100 \%$ methanol for 5 min and stained with $0.5 \%$ crystal violet in $25 \%$ methanol for $10 \mathrm{~min}$. Cells were then washed three times $5 \mathrm{~min}$ each with distilled water to remove excess dye and allowed to dry overnight at room temperature. The incorporated dye was then solubilized in $0.1 \mathrm{M}$ sodium citrate (Sigma-Aldrich) in 50\% ethanol. Next, $100 \mu 1$ of experimental samples were transferred to 96 -well plates and optical densities read at $540 \mathrm{~nm}$ using a xMark microplate absorbance spectrophotometer (Bio-Rad Laboratories, Hercules, CA, USA). For combinatorial experiments glioblastoma cells were also first treated for 6 days with control non-targeting siRNAs or siRNAs targeting MACF1, as previously described. Next $2.5 \times 10^{4}$ siRNA treated cells were replated in 12-well plates in the absence of siRNAs. Controls and conditions for combinatorial experiments included: replated siRNA treated cells grown for $72 \mathrm{~h}$, replated siRNA treated cells exposed to TMZ or DMSO $6 \mathrm{~h}$ after replating and allowed to incubate in TMZ or DMSO for $72 \mathrm{~h}$, and non-treated siRNA cells exposed to TMZ or DMSO $6 \mathrm{~h}$ after plating and grown for $72 \mathrm{~h}$ in the presence of TMZ or DMSO. DMSO is a solvent used to dissolve drugs and was used as a vehicle control to discern the effects of TMZ from the drug solvent. To evaluate the combinatorial effects of silencing MACF1 and TMZ treatment on cell proliferation and viability the cell proliferation assay, as described above, was also used at the end of the 72-h incubation time-point.

Cell motility. Motility assays were conducted according to the manufacturer's instructions (Cell Biolabs, Inc., San Diego, CA, USA). MACF1 silencing was performed as described above. Cell suspensions containing $0.5-1.0 \times 10^{6}$ cells $/ \mathrm{ml}$ of siRNA 


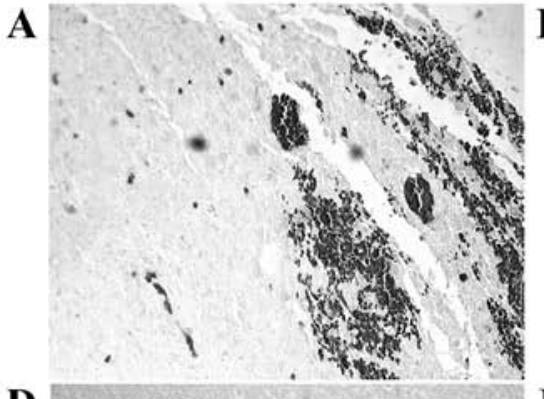

D

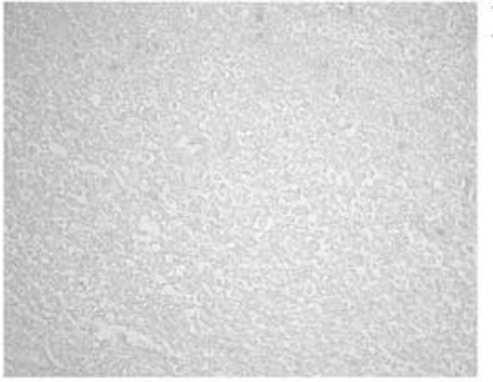

B

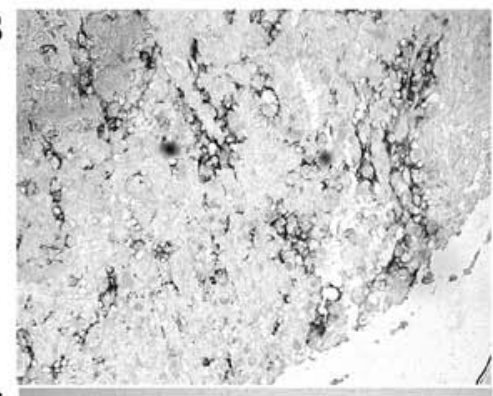

E

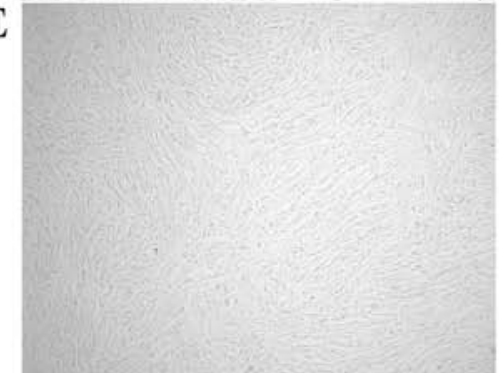

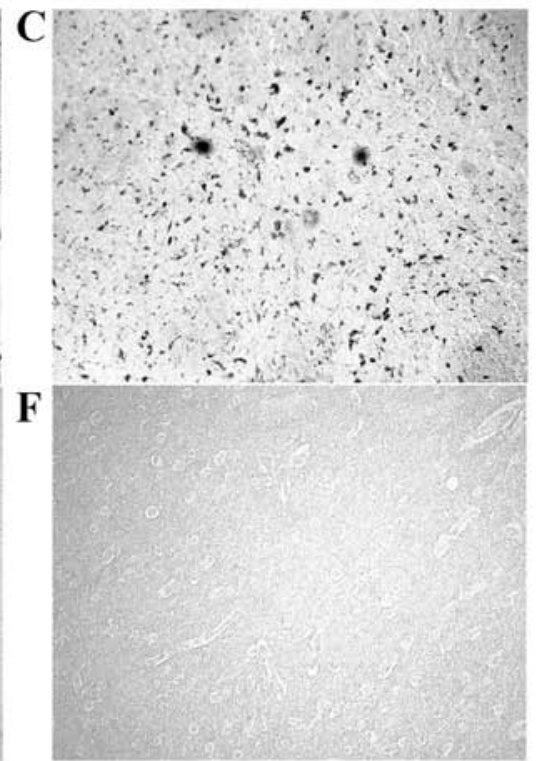

Figure 1. MACF1 expression in brain tumors and normal brain tissue. MACF1 was robustly expressed in glioblastoma (A-C) but not detected in oligodendroglioma (D), medulloblastoma (E), or normal brain tissue (F). MACF1 protein is labeled as black diaminobenzidine (DAB) positive cells. Displayed is an immunohistochemistry labeling experiment representative of three independent experiments that showed similar results (total magnification, $\mathrm{x} 200$ ).

control or siRNA targeted MACF1 treated cells were prepared in serum-free media, while $500 \mu \mathrm{l}$ of media containing $10 \%$ FBS was added to the lower chamber of the migration plate. A total of $300 \mu \mathrm{l}$ of siRNA treated cell suspensions were then added to the inside of each insert and were allowed to incubate for $24 \mathrm{~h}$ at $37^{\circ} \mathrm{C}$ and $5 \% \mathrm{CO}_{2}$. Subsequently, non-migratory cells were removed from plate inserts (per manufacturer's instructions), migratory cells were stained with crystal violet and the dye eluted as described above. Optical densities were read at $595 \mathrm{~nm}$ using a xMark microplate absorbance spectrophotometer (Bio-Rad Laboratories) and statistical evaluation of the data was performed as described below.

Western blotting. Cells treated for 6 days with control siRNAs or siRNAs targeting MACF1 were rinsed with PBS and lysed with CelLytic M Cell lysis reagent (Sigma-Aldrich). Protein concentrations were subsequently determined using the Bradford method. Proteins were separated by SDS-PAGE in 4-15\% polyacrylamide gels (Bio-Rad Laboratories), then transferred to nitrocellulose membranes. For immunoblotting, nitrocellulose membranes were incubated with MACF1 (Santa Cruz Biotechnology), Axin1 (Cell Signaling Technology, Danvers, MA, USA), phospho $\beta$-catenin (Cell Signaling Technology) and GAPDH (Cell Signaling Technology) antibodies recognizing target proteins overnight at $4^{\circ} \mathrm{C}$. The membranes were then incubated with an HRP-conjugated secondary antibody for $1 \mathrm{~h}$ at room temperature, analyzed by enhanced chemiluminescence (ECL) detection system (Thermo Fisher Scientific, Nashville, TN, USA) and visualized using a UVP BioSpectrum imaging system (UVP, LLC, Upland, CA, USA). Densitometry analysis was performed with the Image Studio Lite software (LI-COR Biosciences, Lincoln, NE, USA).

Statistical analysis. Cell proliferation, migration, time-course and combinatorial experiments were each performed at least three times with duplicate or triplicate samples. Means were determined by averaging duplicate or triplicate samples within each independent experiment. Students t-tests and ANOVA analysis were used to evaluate the significance between the experimental conditions with a P-value of 0.05 and 0.01 , respectively.

\section{Results}

Differential expression of MACF1 in brain tumors. Identification and expression profiling of tumor specific biomarkers are essential for clinical diagnostic and prognostic purposes. Using immunohistochemistry procedures we examined MACF1 expression in low and high grade brain tumors. Our data showed that MACF1 was expressed diffusely and at the periphery in $40 \%$ of grade IV glioblastoma tissue sections as compared to its absence in normal brain tissue (Fig. 1A-C). In contrast to its expression in glioblastoma, MACF1 was absent in lower grade brain tumors (oligodendroglioma and medulloblastoma) and normal brain tissue (Fig. 1D-F), but present in grade II-IV astrocytomas as compared to normal brain tissue (Fig. 2). Complementing data observed in human tissue, immunoblotting procedures of MACF1 in glioblastoma cell lines also displayed significantly higher levels of MACF1 as compared to its expression in normal human astrocyte cells (Fig. 2). Collectively, these data provide evidence that MACF1 is predominately expressed in high grade malignant brain tumors and suggest that it is a potential biomarker and target in these cancers.

Anti-tumorigenic effects of suppressing MACF1 in glioblastoma. MACF1 to date has received no consideration as a target in cancer and associated cell behavior. Using siRNAs to inhibit MACF1 function we assessed the effects of downregulating MACF1 expression on glioblastoma cell proliferation and migration. Western blot experiments demonstrated the 


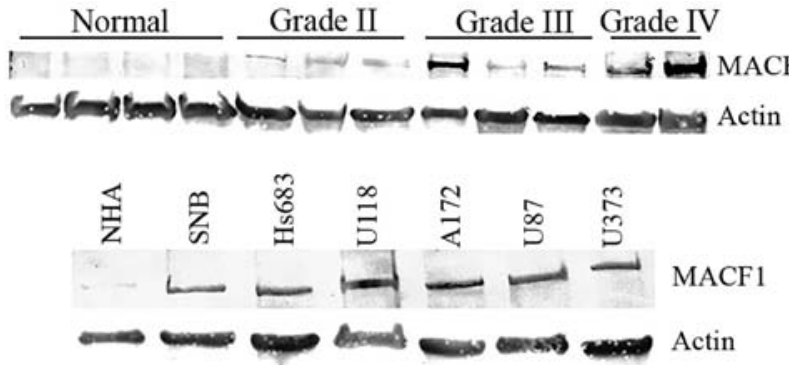

Figure 2. Western blot analysis of MACF1 expression in astrocytomas and glioblastoma cell lines. Astrocytoma tissue taken from tumor patients (top panel) and glioblastoma cell lines (bottom panel) displayed increased MACF1 protein levels as compared to normal human astrocytes (NHA) and normal brain tissue. Immunoblots are representative of at least four experiments that showed equivalent results. Actin was used as a loading control to assess that lanes were loaded with the same amount of total proteins.
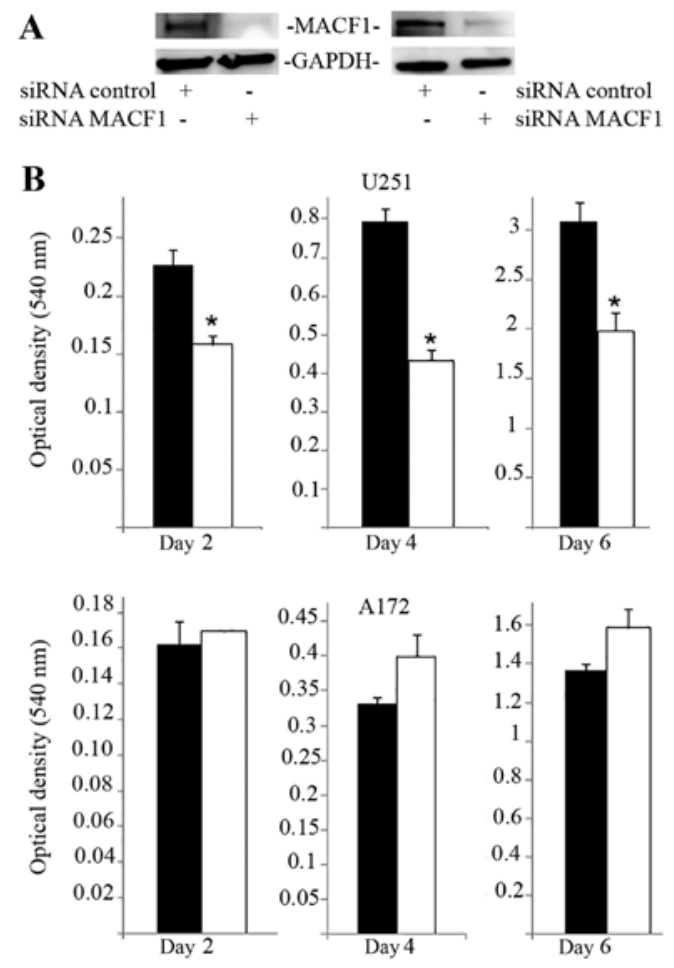

Figure 3. Proliferative response of glioblastoma cells to MACF1 inhibition. (A) Western blot analysis of U251 and A172 cells treated for six days with MACF1 siRNAs nearly abolished MACF1 expression in U251 and A172 cells. (B) Bar graphs show that reduction in MACF1 levels was accompanied with a significant decrease in the proliferative capacity of $\mathrm{U} 251$ cells ( $\mathrm{P}<0.05$ as determined by the Student's t-test; error bars, SE) but not A172 cells. Data shown are representative of at least three independent experiments performed in duplicate. Black bars, control siRNA treated cells; white bars, MACF1 siRNA treated cells.

efficacy of the siRNAs in silencing MACF1 in U251 and A172 glioblastoma cells. Our data also revealed that silencing MACF1 in U251 glioblastoma cells caused a 45 and 37\% decrease in cell viability 4 and 6 days post-treatment, respectively (Fig. 3). However, suppressing MACF1 expression did not reduce A172 glioblastoma cell viability (Fig. 3). We further evaluated the effects of inhibiting MACF1 in patient derived xenograft cell lines (Fig. 4). Time course analysis displayed that shRNAs targeting MACF1 significantly inhibited the
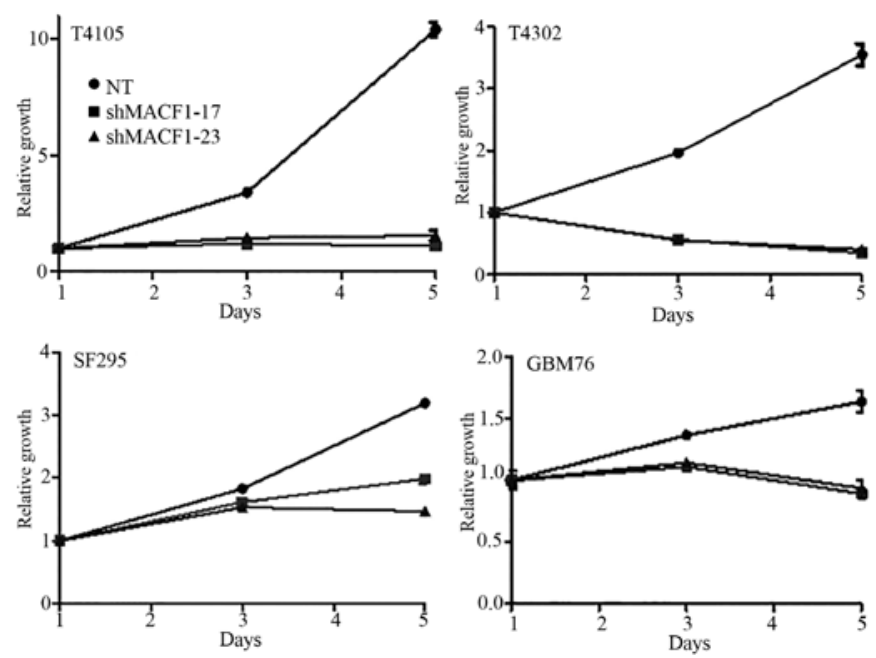

Figure 4. Downregulation of MACF1 impairs proliferation of patient derived xenograft cell lines. Time-course analysis showed that inhibition of MACF1 with shRNAs decreased the proliferative capacity of patient derived glioblastoma cell lines established in xenograft mouse models.
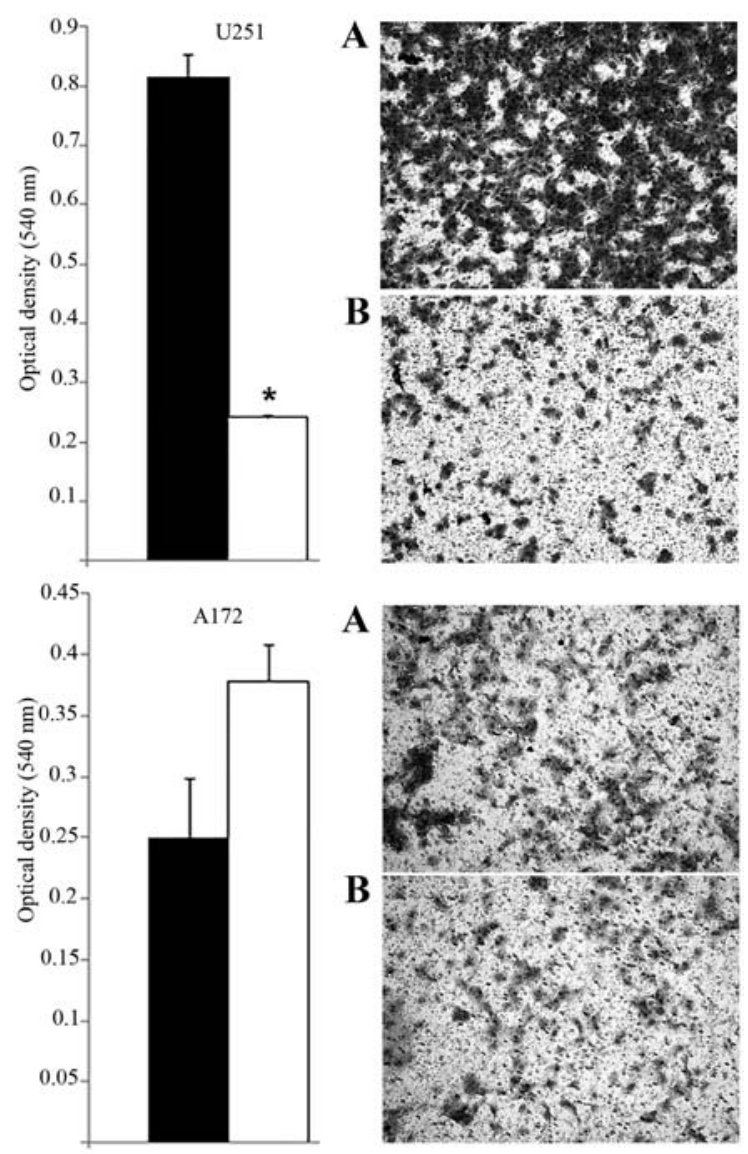

Figure 5. Effects of silencing MACF1 on glioblastoma cell migration. Downregulation of MACF1 significantly impeded cell migration of U251 cells (top panel), but not A172 cells (bottom panel). Bar graphs displayed are representative of three independent experiments performed in duplicate that showed similar results. Black bars, control siRNA treated cells; white bars, MACF1 siRNA treated cells ( $\mathrm{P}<0.05$ as determined by the Student's t-test; error bars, SE).

growth of T4105 and T4302 xenograft cell lines, while SF295 and GBM76 cells were decreased to a much lesser extent 

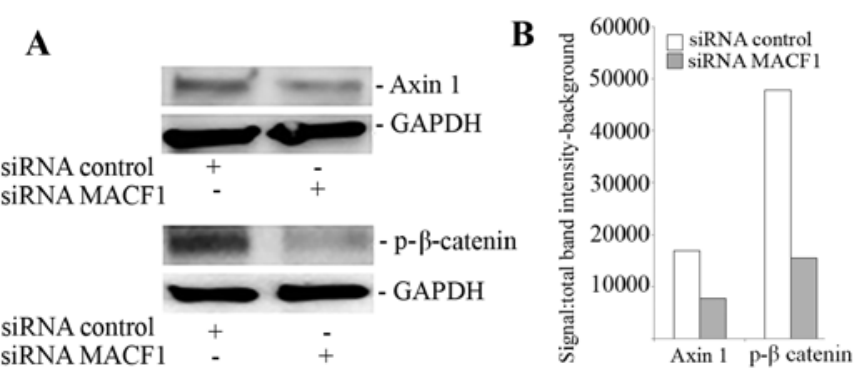

Figure 6. Suppression of MACF1 reduced Axin1 and $\beta$-catenin expression. Inhibition of MACF1 with siRNAs downregulated Axin1 and $\beta$-catenin protein levels in U251 glioblastoma cells. (A) Western blot experiments and (B) densitometric analysis. Data shown are representative of at least three independent experiments that displayed comparative results.
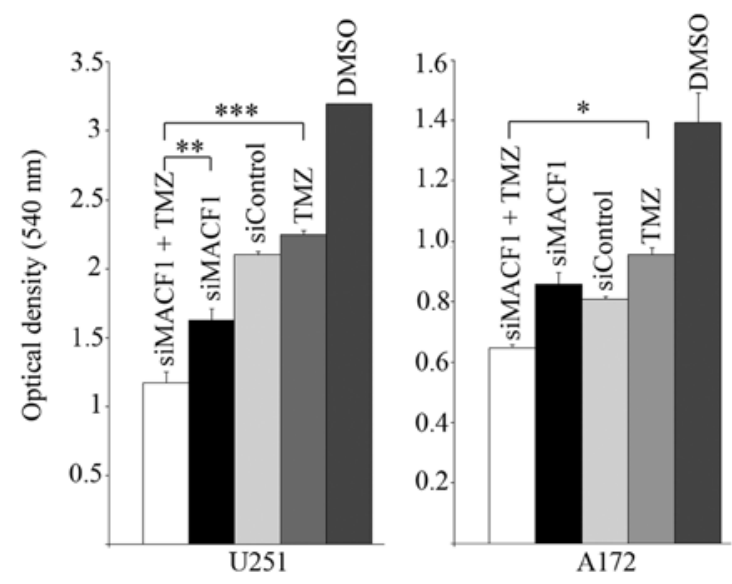

Figure 7. MACF1 repression with concurrent TMZ treatment. Glioblastoma cells with silenced MACF1 treated with $100 \mu \mathrm{M}$ TMZ led to a decrease in glioblastoma cell proliferation compared to the treatment with vehicle DMSO, control siRNAs, TMZ, or MACF1 siRNA alone as determined by ANOVA analysis and Sidak's post hoc multiple comparisons test ( ${ }^{*} \mathrm{P}<0.05$, $\left.{ }^{* *} \mathrm{P}<0.01,{ }^{* * *} \mathrm{P}<0.001\right)$.

(Fig. 4). Consistent with the anti-proliferative effects of downregulating MACF1 protein levels in U251 cells, repression of MACF1 also significantly reduced U251 cell migration but not A172 cells (Fig. 5). Furthermore, because MACF1 has been implicated as a modulatory signaling contributor of the Wnt signaling pathway $(16,17)$, whose dysregulation is well known to be involved in tumor proliferation and migratory invasion in a number of human cancers (18-20) including malignant brain tumors (21-24) we evaluated the impact of silencing MACF1 on Wnt-signaling proteins. Our data showed that downregulation of MACF1 protein levels was accompanied by decreased Axin1 and activated form of $\beta$-catenin protein levels (Fig. 6), suggesting that reduction of these Wnt-signaling mediators contribute to the anti-tumorigenic response of impairing MACF1 in glioblastoma.

Synergistic effects of MACF1 inhibition and TMZ. Chemotherapeutic agents used to treat glioblastoma have been minimally effective in part as a consequence of expressed genetic aberrations that contribute to intrinsic and acquired tumor resistance (25). We subsequently performed combinatorial studies to test the effectiveness of downregulating MACF1

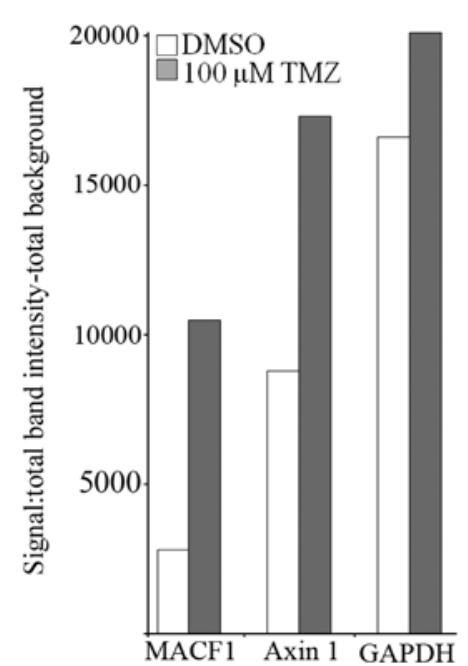

Figure 8. Induction of MACF1 and Axin1 by TMZ. Densitometric analysis of immunoblot showed that TMZ upregulated MACF1 and Axin1 protein levels in U251 glioblastoma cells. Data shown are representative of at least three independent experiments that displayed comparative results.

along with the effects of the alkylating agent, temozolomide (TMZ) in glioblastoma cells. For combinatorial experiments, glioblastoma cells were treated with siRNAs targeting MACF1 and $100 \mu \mathrm{M} \mathrm{TMZ}$ and compared to cells treated with TMZ alone or cells treated with siRNAs targeting MACF1 alone. ANOVA analysis $(\mathrm{P}<0.01)$ of data from cell proliferation assays revealed that the combination of MACF1 and TMZ treatment had the strongest effect on decreasing glioblastoma cell viability when compared to other treatment conditions and controls. Additionally, concurrent treatment of U251 glioblastoma cells with MACF1 targeting siRNAs and TMZ exhibited a 1.5- and 2-fold cell viability decrease as compared to cells treated with either MACF1 siRNAs or TMZ alone (Fig. 7). A similar response was observed in A172 cells, which displayed a 1.32- and 1.48-fold decrease in cell viability when treated concurrently with TMZ and siRNAs targeting MACF1 as compared to treatment with either of these conditions alone. We further examined by western blotting the effects of TMZ on the expression levels of MACF1 and Axin1 in U251 cells treated for $3 \mathrm{~h}$ with $100 \mu \mathrm{M}$ TMZ. These experiments showed that TMZ increased the protein levels of MACF1 and the Wnt-cytoplasmic complex protein, Axin1 in U251 cells (Fig. 8), further supporting the combinatorial approach and need to inhibit MACF1 in glioblastoma cells treated with TMZ to achieve a synergistic effect.

\section{Discussion}

The scope of genetic abnormalities in the glioblastoma genome was recently supported in a broad genome sequence study that revealed 512 mutated genes in U87 glioblastoma cells, the most commonly studied glioblastoma in vitro cell line model (26). This study provided perspective on the instability of the glioblastoma genome and on the scope of functional studies that must be undertaken to decipher the roles of uncharacterized genetic aberrations and their protein products in glioblastoma.

Cytoskeletal proteins play a key role in motility, invasion and proliferation (27). In this study we investigated the 
cytoskeletal spectraplakin protein, MACF1, as a potential diagnostic and prognostic marker and have evaluated its potential to be a target for glioblastoma therapy. Our findings that MACF1 was predominantly expressed in glioblastoma but absent in normal brain tissue and lower grade brain tumors suggest that MACF1 is a potential tumor-specific marker of this grade IV brain tumor. Taken together, our finding that MACF1 downregulation with RNA interference reduces the proliferation and migration of glioblastoma cells, indicates that MACF1 expression positively influences the proliferation and migration of glioblastoma cells. A similar conclusion was reached in studies by $\mathrm{Hu}$ et al (28) and $\mathrm{Wu}$ et al (17) which demonstrated that genetic inhibition and absence of MACF1 causes a cell cycle arrest associated decrease in osteoblastic cell proliferation and impaired keratinocyte migration, respectively. It should also be mentioned that the role of cell death was not examined as an underlying cause of the decrease in glioblastoma cell proliferation and viability in response to negatively regulating MACF1 function in this study, which is complicated by the established evasiveness of glioblastomas from apoptotic cell death in response to various treatment modalities. In this regard forthcoming studies will examine whether inhibition of MACF1 promotes apoptotic cell death or autophagy, the preferred mode of cell death in glioblastomas.

Along with its influence on cell behavior, an early investigation by Chen et al (16) also revealed that MACF1 was involved in the Wnt-signaling axis and showed that downregulation of MACF1 decreased nuclear $\beta$-catenin and led to an inhibition of Wnt induced $\beta$-catenin-dependent transcriptional activation in P19 and Rat-1 cells. Paralleling these observations, our results demonstrate that the scaffolding protein Axin1 and the transcriptional activator $\beta$-catenin of the Wnt signaling pathway are decreased in cells with suppressed MACF1. These data indicate that MACF1 contributes to the regulation of glioblastoma cell proliferation and migration by intervening in the Wnt-signaling pathway.

Our results also show that although MACF1 is expressed at similar levels in U251 and A172 glioblastoma cells, its silencing impedes motility and proliferation in U251, but not A172 cells. This could be due to heterogeneous genetic alterations between these two cell lines, such as p53 which is mutated in U251 and wild-type in A172 or differential expression of MACF1 isoforms. In support of this hypothesis, glioblastoma cell lines have been shown to be disparate in their genetic alterations (29). In addition, several MACF1 isoforms with different 5'UTRs and variations in the N-terminal actin-binding domain region have been described (30), but their functions have yet to be deciphered. Furthermore, it has been demonstrated that isoform specific silencing of the actin-binding protein $\alpha$-actinin in glioblastoma cells has been shown to lead to different cellular outcomes (31), raising the possibility that differential expression of MACF1 isoforms may affect glioblastoma cell behavior. To this end, future studies will investigate the inhibitory effects of silencing different MACF1 isoforms that could impair glioblastoma cell proliferation and migration, particularly in A172 cells that were impervious to MACF1 inhibition in this study.

Clinically, TMZ is among the lead chemotherapeutic agents used for the treatment of nervous system cancers, particularly malignant brain tumors, because of its bioavail- ability and limited toxicity (32). However, a caveat of its efficacy has been intrinsic and acquired resistance as a result in part of activating pro-survival signaling mediators (33-35). From a therapeutic precision medicine standpoint, our data show that in glioblastoma cells in which MACF1 downregulation decreases proliferation and motility, reduced MACF1 levels also enhances the anti-proliferative effect of TMZ. Interestingly, our results also show that TMZ induces the expression of MACF1 and Axin1, indicating that MACF1-Wnt signaling, may contribute to TMZ resistance and perpetuate glioblastoma cell survival. These data are in line with findings that combinatorial targeted therapy approaches with TMZ is efficient at enhancing the progression-free survival of patients with genetically diverse glioblastomas (36-38). Taken together, our results identify MACF1 as a unique target with the potential to broaden combinatorial treatment paradigms in glioblastomas with diverse genetic profiles.

\section{Acknowledgements}

The present study was supported by the Tennessee State University Department of Biology and Division of Research and Sponsored Programs.

\section{References}

1. Ostrom QT, Bauchet L, Davis FG, Deltour I, Fisher JL, Langer CE, Pekmezci M, Schwartzbaum JA, Turner MC, Walsh KM, et al: The epidemiology of glioma in adults: A 'state of the science' review. Neuro Oncol 16: 896-913, 2014.

2. Reardon DA and Wen PY: Glioma in 2014: Unravelling tumour heterogeneity - implications for therapy. Nat Rev Clin Oncol 12: 69-70, 2015.

3. Parsons DW, Jones S, Zhang X, Lin JC, Leary RJ, Angenendt P, Mankoo P, Carter H, Siu IM, Gallia GL, et al: An integrated genomic analysis of human glioblastoma multiforme. Science 321: 1807-1812, 2008.

4. Verhaak RG, Hoadley KA, Purdom E, Wang V, Qi Y, Wilkerson MD, Miller CR, Ding L, Golub T, Mesirov JP, et al; Cancer Genome Atlas Research Network: Integrated genomic analysis identifies clinically relevant subtypes of glioblastoma characterized by abnormalities in PDGFRA, IDH1, EGFR, and NF1. Cancer Cell 17: 98-110, 2010.

5. Heimberger AB, Hlatky R, Suki D, Yang D, Weinberg J, Gilbert M, Sawaya R and Aldape K: Prognostic effect of epidermal growth factor receptor and EGFRvIII in glioblastoma multiforme patients. Clin Cancer Res 11: 1462-1466, 2005.

6. Pelloski CE, Ballman KV, Furth AF, Zhang L, Lin E, Sulman EP, Bhat K, McDonald JM, Yung WK, Colman H, et al: Epidermal growth factor receptor variant III status defines clinically distinct subtypes of glioblastoma. J Clin Oncol 25: 2288-2294, 2007.

7. Hegi ME, Diserens AC, Gorlia T, Hamou MF, de Tribolet N, Weller M, Kros JM, Hainfellner JA, Mason W, Mariani L, et al: MGMT gene silencing and benefit from temozolomide in glioblastoma. N Engl J Med 352: 997-1003, 2005.

8. Rivera AL, Pelloski CE, Gilbert MR, Colman H, De La Cruz C, Sulman EP, Bekele BN and Aldape KD: MGMT promoter methylation is predictive of response to radiotherapy and prognostic in the absence of adjuvant alkylating chemotherapy for glioblastoma. Neuro Oncol 12: 116-121, 2010.

9. Sanchez-Soriano N, Travis M, Dajas-Bailador F, GonçalvesPimentel C, Whitmarsh AJ and Prokop A: Mouse ACF7 and drosophila short stop modulate filopodia formation and microtubule organisation during neuronal growth. J Cell Sci 122: 2534-2542, 2009.

10. Munemasa Y, Chang CS, Kwong JM, Kyung H, Kitaoka Y, Caprioli J and Piri N: The neuronal EGF-related gene Nell2 interacts with Macf1 and supports survival of retinal ganglion cells after optic nerve injury. PLoS One 7: e34810, 2012. 
11. Bernier G, Pool M, Kilcup M, Alfoldi J, De Repentigny Y and Kothary R: Acf7 (MACF) is an actin and microtubule linker protein whose expression predominates in neural, muscle, and lung development. Dev Dyn 219: 216-225, 2000.

12. Sjöblom T, Jones S, Wood LD, Parsons DW, Lin J, Barber TD, Mandelker D, Leary RJ, Ptak J, Silliman N, et al: The consensus coding sequences of human breast and colorectal cancers. Science 314: 268-274, 2006.

13. Misquitta-Ali CM, Cheng E, O'Hanlon D, Liu N, McGlade CJ, Tsao MS and Blencowe BJ: Global profiling and molecular characterization of alternative splicing events misregulated in lung cancer. Mol Cell Biol 31: 138-150, 2011.

14. Carlson BL, Pokorny JL, Schroeder MA and Sarkaria JN: Establishment, maintenance and in vitro and in vivo applications of primary human glioblastoma multiforme (GBM) xenograft models for translational biology studies and drug discovery. Curr Protoc Pharmacol Chapter 14: 16, 2011.

15. Chiba K, Kawakami K and Tohyama K: Simultaneous evaluation of cell viability by neutral red, MTT and crystal violet staining assays of the same cells. Toxicol In Vitro 12: 251-258, 1998.

16. Chen HJ, Lin CM, Lin CS, Perez-Olle R, Leung CL and Liem RK The role of microtubule actin cross-linking factor 1 (MACF1) in the Wnt signaling pathway. Genes Dev 20: 1933-1945, 2006.

17. Wu X, Shen QT, Oristian DS, Lu CP, Zheng Q, Wang HW and Fuchs E: Skin stem cells orchestrate directional migration by regulating microtubule-ACF7 connections through GSK3 $\beta$. Cell 144: 341-352, 2011.

18. Yin X, Xiang T, Li L, Su X, Shu X, Luo X, Huang J, Yuan Y, Peng W, Oberst M, et al: DACT1, an antagonist to Wnt/ $\beta$-catenin signaling, suppresses tumor cell growth and is frequently silenced in breast cancer. Breast Cancer Res 15: R23, 2013.

19. Narayanan BA, Doudican NA, Park J, Xu D, Narayanan NK, Dasgupta R and Mazumder A: Antagonistic effect of smallmolecule inhibitors of Wnt $/ \beta$-catenin in multiple myeloma. Anticancer Res 32: 4697-4707, 2012.

20. Bakker ER,Das AM,Helvensteijn W,Franken PF, Swagemakers S van der Valk MA, ten Hagen TL, Kuipers EJ, van Veelen W and Smits R: Wnt5a promotes human colon cancer cell migration and invasion but does not augment intestinal tumorigenesis in Apc1638N mice. Carcinogenesis 34: 2629-2638, 2013.

21. Yu JM, Jun ES, Jung JS, Suh SY, Han JY, Kim JY, Kim KW and Jung JS: Role of Wnt5a in the proliferation of human glioblastoma cells. Cancer Lett 257: 172-181, 2007.

22. Pu P, Zhang Z, Kang C, Jiang R, Jia Z, Wang G and Jiang H: Downregulation of Wnt 2 and beta-catenin by siRNA suppresses malignant glioma cell growth. Cancer Gene Ther 16: 351-361, 2009.

23. De Robertis A, Valensin S, Rossi M, Tunici P, Verani M, De Rosa A, Giordano C, Varrone M, Nencini A, Pratelli C, et al: Identification and characterization of a small-molecule inhibitor of Wnt signaling in glioblastoma cells. Mol Cancer Ther 12: 1180-1189, 2013

24. Kaur N, Chettiar S, Rathod S, Rath P, Muzumdar D, Shaikh ML and Shiras A: Wnt3a mediated activation of $\mathrm{Wnt} / \beta$-catenin signaling promotes tumor progression in glioblastoma. Mol Cell Neurosci 54: 44-57, 2013
25. Ramirez YP, Weatherbee JL, Wheelhouse RT and Ross AH: Glioblastoma multiforme therapy and mechanisms of resistance. Pharmaceuticals (Basel) 6: 1475-1506, 2013.

26. Clark MJ, Homer N, O'Connor BD, Chen Z, Eskin A, Lee H, Merriman B and Nelson SF: U87MG decoded: The genomic sequence of a cytogenetically aberrant human cancer cell line. PLoS Genet 6: e1000832, 2010.

27. Quick Q, Paul M and Skalli O: Roles and potential clinical applications of intermediate filament proteins in brain tumors. Semin Pediatr Neurol 22: 40-48, 2015.

28. Hu L, Su P, Li R, Yan K, Chen Z, Shang P and Qian A: Knockdown of microtubule actin crosslinking factor 1 inhibits cell proliferation in MC3T3-E1 osteoblastic cells. BMB Rep 48: 583-588, 2015.

29. Ishii N, Maier D, Merlo A, Tada M, Sawamura Y, Diserens AC and Van Meir EG: Frequent co-alterations of TP53, p16/ $C D K N 2 A, p 14^{A R F}$, PTEN tumor suppressor genes in human glioma cell lines. Brain Pathol 9: 469-479, 1999.

30. Hu L, Su P, Li R, Yin C, Zhang Y, Shang P, Yang T and Qian A: MACF1, a versatile spectraplakin: Isoforms, unique structures, and functions. BMB Rep 49: 37-44, 2016.

31. Quick Q and Skalli O: Alpha-actinin 1 and alpha-actinin 4: Contrasting roles in the survival, motility, and RhoA signaling of astrocytoma cells. Exp Cell Res 316: 1137-1147, 2010.

32. von Neubeck C, Seidlitz A, Kitzler HH, Beuthien-Baumann B and Krause M: Glioblastoma multiforme: Emerging treatments and stratification markers beyond new drugs. Br J Radiol 88: 20150354, 2015

33. Loftus JC, Dhruv H, Tuncali S, Kloss J, Yang Z, Schumacher CA, Cao B, Williams BO, Eschbacher JM, Ross JT, et al: TROY (TNFRSF19) promotes glioblastoma survival signaling and therapeutic resistance. Mol Cancer Res 11: 865-874, 2013.

34. Choi EJ, Cho BJ, Lee DJ, Hwang YH, Chun SH, Kim HH and Kim IA: Enhanced cytotoxic effect of radiation and temozolomide in malignant glioma cells: Targeting PI3K-AKT-mTOR signaling, HSP90 and histone deacetylases. BMC Cancer 14: 17, 2014.

35. Vo VA, Lee JW, Lee HJ, Chun W, Lim SY and Kim SS: Inhibition of JNK potentiates temozolomide-induced cytotoxicity in U87MG glioblastoma cells via suppression of Akt phosphorylation. Anticancer Res 34: 5509-5515, 2014.

36. Hainsworth JD, Shih KC, Shepard GC, Tillinghast GW, Brinker BT and Spigel DR: Phase II study of concurrent radiation therapy, temozolomide, and bevacizumab followed by bevacizumab/everolimus as first-line treatment for patients with glioblastoma. Clin Adv Hematol Oncol 10: 240-246, 2012.

37. Chinot OL, Wick W, Mason W, Henriksson R, Saran F, Nishikawa R, Carpentier AF, Hoang-Xuan K, Kavan P, Cernea D, et al: Bevacizumab plus radiotherapy-temozolomide for newly diagnosed glioblastoma. N Engl J Med 370: 709-722, 2014

38. Clarke JL, Molinaro AM, Phillips JJ, Butowski NA, Chang SM, Perry A, Costello JF, DeSilva AA, Rabbitt JE and Prados MD: A single-institution phase II trial of radiation, temozolomide, erlotinib, and bevacizumab for initial treatment of glioblastoma. Neuro Oncol 16: 984-990, 2014. 